\title{
Simulación: Una herramienta útil en la formación quirúrgica moderna*
}

\author{
Simulation: Teaching surgical skills-changes \\ Drs. ENRIQUE LANZARINI S. ${ }^{1}$, VALERIA SCHONSTEDT P. ${ }^{2}$, MARIO ABEDRAPO M.1, \\ JULIO YARMUCH G. ${ }^{1}$, ATTILA CSENDES J. ${ }^{1}$, ALBERTO RODRÍGUEZ N. ${ }^{1}$ \\ ${ }^{1}$ Departamento de Cirugía, Hospital Clínico Universidad de Chile \\ 2Interna Facultad de Medicina Universidad de Chile, Santiago. Chile
}

Ya a fines del siglo XIX se describen en Europa, por primera vez de forma metódica y bien estructurada, métodos de formación y entrenamiento para el desarrollo de habilidades quirúrgicas. Los cirujanos en formación debían realizar cirugías que progresaban en complejidad, siempre bajo la tutela de un cirujano experto, lo cual es recordado y ejemplificado con la frase "vea uno, haga uno, enseñe uno". Este sistema parecía ofrecer buenos resultados; sin embargo, la revolución que ha significado en los últimos años el desarrollo de la laparoscopia, en que la cirugía ha aumentado su grado de tecnología y en que ha existido un aumento en la protección de los pacientes, es necesario un cambio en la formación del cirujano. Aprender estas técnicas, requiere desarrollar nuevas habilidades y destrezas para manejar ciertas características propias como son la falta de percepción táctil directa, la mayor coordinación de movimientos complejos y la pérdida de percepción de profundidad $^{1-3}$.

El aprendizaje de estas habilidades técnicas en pacientes, resulta cada vez más restringido por presiones del medio, siendo principalmente éticas, es decir, tratando de poner en el mínimo riesgo a los pacientes. Esto ha impulsado el desarrollo de un nuevo modelo de adquisición de habilidades quirúrgicas, con el cual el cirujano en formación incorpora la técnica básica de laparoscopia me- diante simuladores, lo que le permite obtener las habilidades quirúrgicas mínimas para un óptimo enfrentamiento de los pacientes en el pabellón. Se basan en la teoría sobre la adquisición de las habilidades motoras de Fitts y Posner, que consta de 3 etapas ${ }^{4}$.

La primera es la etapa cognitiva, en la cual se le explica y demuestra al cirujano practicante la ejecución de los elementos básicos de una cirugía, como por ejemplo, hacer un nudo. Para esto el cirujano debe pensar en la ejecución, paso a paso, de dichas acciones: cómo tomar los hilos, cómo cruzarlos, cómo realizar el nudo, etc. Posteriormente le sigue la etapa integrativa, en la cual el aprendiz debe incorporar la técnica aprendida; para esto, el cirujano debe repetir múltiples veces la tarea motora a realizar. Y por último, se describe la etapa autónoma, en que la actividad motora es realizada de forma fluida, sin requerir pensar en su ejecución, logrando un desempeño sin errores y en forma continua.

El objetivo del entrenamiento con simuladores es lograr el aprendizaje de las dos primeras etapas previo al contacto con los pacientes, logrando un automatismo de la técnica operatoria básica para que, cuando el cirujano deba participar activamente en el quirófano, pueda concentrarse en el desarrollo global de la cirugía, y no en sus componentes elementales. Por otro lado, si el cirujano en forma-

*Recibido el 22 de Noviembre de 2007 y aceptado para publicación el 23 de Diciembre de 2007.

Correspondencia: Dr. Alberto Rodríguez N.

Santos Dumont 999, Santiago, Chile

e-mail: alrodrigu@med.uchile.cl 
ción ya maneja estas técnicas, el cirujano experto puede instruirlo durante la cirugía en relación al procedimiento en particular, y no a detalles de la técnica básica.

Esta manera de enseñar presenta múltiples beneficios, entre los cuales destacan: una erradicación del uso de los pacientes como una plataforma de enseñanza, optimización y ahorro de recursos del quirófano (al reducirse la duración del procedimiento), disminución de mal uso del instrumental y, por otro lado, una posible reducción en demandas legales por mal praxis ${ }^{4,5}$. Con esto se logra un ambiente seguro de entrenamiento para los pacientes, para el cirujano en formación y para el cirujano docente.

En la formación de cirujanos, el ensayo y la experiencia determinan la calidad del desempeño de éste. Sin embargo, se ha visto que el volumen de práctica quirúrgica no es el determinante más importante, sino que juega un rol fundamental la calidad del ejercicio realizado. Una práctica a conciencia, focalizando el desarrollo de determinadas habilidades, bajo supervisión y con un sistema de corrección, aporta un aprendizaje mucho más eficaz que aquellas prácticas no supervisadas. Esto sólo se puede lograr fuera del quirófano, ya que durante la cirugía, dado las presiones que ésta conlleva y su alta complejidad, resulta difícil para el cirujano en formación concentrarse únicamente en aspectos de su técnica.

Como mencionamos anteriormente, el ensayo en pacientes es cada vez menos recomendable, tanto por implicancias ético-legales, como por la disponibilidad del quirófano que requiere y por no presentar las condiciones adecuadas para un entrenamiento óptimo. Ziv ha planteado que en nuestro tiempo la simulación debería ser considerada un imperativo ético, ya que los pacientes deben ser protegidos como sea posible y no deben utilizarse como instrumento de aprendizaje ${ }^{6}$. Razones como esta han impulsado la creación de diversos métodos inanimados para el desarrollo de destrezas laparoscópicas.

Los sistemas más básicos, corresponden a las cajas de entrenamiento, ya sea con visión directa, a través de espejos o mediante filmación con cámaras de video ${ }^{7}$. En cambio, los sistemas más avanzados - simuladores de realidad virtual - reportados desde el año 1996, corresponden a programas de computación que reproducen una laparoscopia, proporcionándole al cirujano una sensación táctil y de manipulación de tejidos similar a la realidad. Esto se logra mediante dispositivos de realimentación de fuerza, denominados dispositivos hápticos. Además, este sistema tiene la capacidad de evaluar la destreza del cirujano frente a determinados procedimientos (corte, disección, prehensión) lo que posibilita objetivar destrezas.

Los más recientes simuladores virtuales, los de tercera generación, además de permitir la interacción física con cada estructura anatómica, como la deformación del tejido, consideran la naturaleza funcional de los órganos. Por ejemplo, cortar vaso sanguíneo importante tiene influencias en la presión sanguínea, repercutiendo en los demás órganos, o lesiones tumorales modifican las propiedades del tejido en cuestión ${ }^{8}$. Hacemos especial mención del simulador laparoscópico LapMentor TM (Simbionix USA Corp., Cleveland, OH), que se encuentra disponible en nuestro Departamento de Cirugía, para el entrenamiento y la formación de cirujanos (Figuras 1 y 2). Es un equipo de alta fidelidad que presenta el descrito sistema háptico,

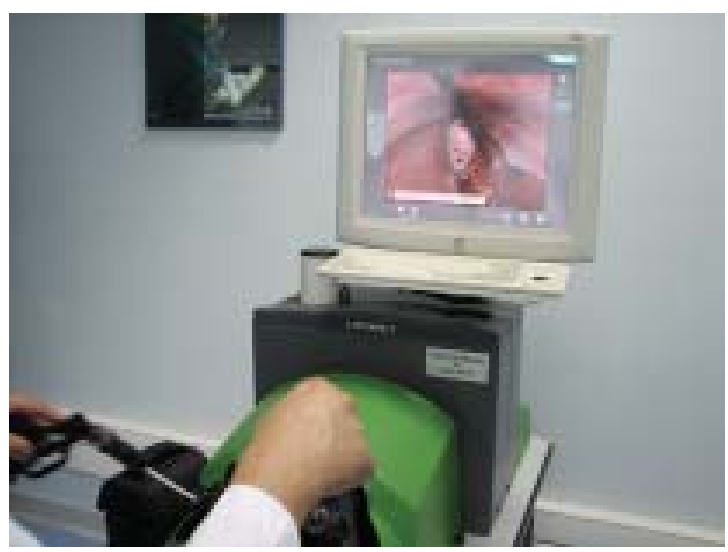

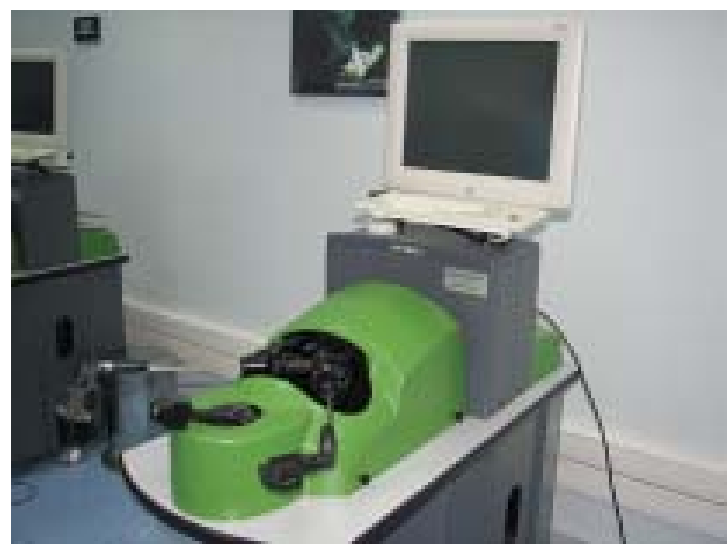

Figura 1 y 2. LapMentor TM. Simulador de Cirugía Laparoscópica. Centro de Alta Tecnología Henry Mayer, Hospital Clínico
Universidad de Chile. Universidad de Chile. 
confiriéndole resistencia a los tejidos al ser manipulados. Además, posee un sistema de retroalimentación, otorgándole al cirujano una evaluación de su desempeño mediante la medición del tiempo que requirió, evaluando la precisión de los ejercicios, la eficiencia de los movimientos, y el cumplimiento de parámetros de seguridad ejercidos ${ }^{9}$.

En el año 2006, un estudio aleatorio, doble ciego, realizado por el Departamento de Cirugía de la Universidad de Michigan, comprobó que residentes de distintas especialidades quirúrgicas que fueron entrenados únicamente en una oportunidad con LapMentor ${ }^{\mathrm{TM}}$ presentaron un considerable mejor desempeño, en comparación a aquellos que no recibieron entrenamiento ${ }^{9}$.

Todas estas ventajas, parecen colocan a los simuladores como piedra angular en el entrenamiento de técnicas endoscópicas y laparoscópicas. Pese a esto, no todos los estudios han sido concluyentes con respecto a la demostración de su eficacia.

En suma, los beneficios de la simulación laparoscópica como herramienta de entrenamiento en la formación de cirujanos parecen no ser discutibles: el desarrollo de habilidades quirúrgicas fuera del quirófano permite aprender aquellas maniobras básicas de cirugía, permitiendo repeticiones tantas veces como sea necesario, sin la presión propia de la cirugía y logrando tomar conciencia de los movimientos ejercidos, sin riesgos para el paciente y sin demora en la utilización de los recintos quirúrgicos, permitiendo optimizar en éstos la atención del practicante en el desarrollo global de la cirugía y las enseñanzas de los cirujanos expertos.

Sin embargo, es indiscutible el requerimiento de más estudios que demuestren los beneficios de estos sistemas a gran escala. Es importante recalcar que en un futuro, cada nuevo simulador que sea elaborado, debe necesariamente ser validado tanto en su función como herramienta para el entrenamiento quirúrgico, como para evaluación del desempeño de los cirujanos.

Como idea final, hay que destacar que con todo este desarrollo educacional, el fin último es proteger lo más posible a nuestros pacientes y realizar una enseñanza de las nuevas técnicas quirúrgicas de una manera más eficiente y de mejor calidad, en un ambiente seguro para todos los involucrados en esta importante tarea para nuestra sociedad.

\section{REFERENCIAS}

1. Justo-Janeiro J, Pedroza- Melendez A, Prado E, Theurel-Vincent G, Vazquez-de Lara L. Un nuevo simulador de laparoscopia. Cir Ciruj 2007; 75: 1923.

2. Rodríguez G, Mendia E, Fresneda P, Montero J, Correa C, Fresneda V. Herramientas de Aprendizaje en Técnicas Endoscópicas Minimamente Invasivas. Servicio de Cirugía General y del Aparato Digestivo. Hospital Universitario Ramón y Cajal. Madrid. www.seclaendosurgery.com/seclan19/tecno. htlm. Consultado el 23 Oct 2007.

3. Monserrat C, Alcañiz M, Meier U, Poza J, Juan M, Grau V. Simulador para el entrenamiento en cirugías avanzadas. Universidad Politécnica de Valencia. www.dsic.upv.es/ cmonserr/Articulos/AA028 pdf. Consultado el 23 Oct 2007.

4. Reznick RK, MacRae H. Teaching surgical skillschanges in the wind. N Engl J Med. 2006; 355: 2664-2669.

5. Aggarwal R, Darzi A. Technical-skills training in the 21st Century. N Engl J Med. 2006; 355: 2695-2696.

6. Ziv A, Wolpe PR, Small SD. Simulation-based medical education: an ethical imperative. Acad Med 2003; 78: 783-788.

7. Justo J. Simuladores para Cirugía Endoscópica. Asociación Mexicana de Cirugía Endoscopica. 2005; 6: 115-120.

8. Sánchez F, Gómez E, Pagador J, Monserrat C, Sánchez-Gijón S, Alcañiz M. et al. Integración de la Tecnología de Simulación Quirúrgica en el Programa de Aprendizaje de Cirugía de Mínima Invasión. www.conganat.org/seis/is/is47/IS47_09 pdf. Consultado el 23 Oct 2007.

9. Andreatta PB, Woodrum DT, Birkmeyer JD, Yellamanchilli RK, Doherty GM, Gauger PG. Laparoscopic with LapMentor ${ }^{\mathrm{TM}}$ training: results of a randomized, double-blinded study. Ann Surg. 2006; 243: 854-860.

10. Sutherland L, Middleton P, Anthony A, Hamdorf J, Cregan P, Scott D. et al. Surgical simulation: a systematic review. Ann Surg. 2006; 243: 291-300. 